\title{
Anisotropic properties of riddled basins
}

\author{
Peter Ashwin*and Michael Breakspear ${ }^{\dagger}$ \\ Department of Mathematics and Statistics \\ University of Surrey \\ Guildford GU2 7XH, UK.
}

September 8, 2000

\begin{abstract}
We consider some general properties of chaotic attractors with riddled basins of attraction (basins with positive measure but open dense complements) in dynamical systems with symmetries. We investigate how a basin of attraction can riddled in some directions and not in others if the attractor is contained in the intersection of several invariant subspaces. This means that the extreme sensitivity to added noise (bubbling) associated with riddled basin attractors is in fact strongly dependent on the nature of the noise. We discuss examples of this for systems of globally coupled maps.
\end{abstract}

\section{Introduction}

Suppose that $f: M \rightarrow M$ is a smooth map of some $M=\mathbf{R}^{m}$ to itself. If $A \subset M$ is a compact invariant set, we define the basin of $A$ to be the set of points whose $\omega$-limit sets are contained within $A$; i.e. $\mathcal{B}(A)=\{x \in M: \omega(x) \subset A\}$. We say $A$ is an attractors if $\mathcal{B}(A)$ is large in some sense; either an open set (as for example in the case of asymptotically stable attractors) or if $\mathcal{B}(A)$ has positive $m$-dimensional Lebesgue measure (in the case of Milnor attractors [12]).

If an attractor has a basin that has positive measure but there are no open sets possessing full measure subsets contained in the basin, then the basin is said to be riddled. In a remarkable paper [1] it was shown not only that there exist dynamical systems with invariant subspaces but also that these are in some sense 'typical' for dynamics with invariant subspaces. For systems possessing symmetries, such invariant subspaces are forced to exist and so riddled basins are be common for chaotic attractors in symmetric systems, especially for attractors that are have lost asymptotic stability but that are still Milnor attractors.

*Address from Oct 2000: School of Mathematical Sciences, Laver Building, University of Exeter, Exeter EX4 4QJ, UK.

${ }^{\dagger}$ Also: Brain Dynamics Centre, Dept of Psychological Medicine, Westmead Hospital, Sydney, NSW 2145, Australia. 
The study of such riddled basin attractors and related phenomena has been undertaken from theoretical and numerical viewpoints $[1,4,11,16,20]$ mostly using transverse Lyapunov exponents and skew-product models. In particular the bifurcations to riddled basins $[14,10]$, the sensitivity of such attractors to noise (bubbling) $[3,19]$ and the distinction of local and global riddling have been studied by several researchers $[4,11,5]$.

In this paper we turn our attention to properties of riddling that can appear in the presence of more than one invariant subspaces; we observe that riddling may occur in some directions but not others, we say that riddling is typically anisotropic in transverse directions and this gives rise to anisotropic sensitivity to noise. We show there are internal riddling transitions where the number of directions in which riddling occurs changes. This is supported with some observations from numerical simulations of coupled map systems.

\subsection{Symmetries of maps}

We consider a smooth iterated mapping $f: M \rightarrow M$ for $M=\mathbf{R}^{m}$ some Euclidean space. We write $\ell_{N}($.$) to denote Lebesgue measure on N$ any linear subspace of $M$. Suppose that $M=\mathbf{R}^{m}$ commutes (is equivariant) with the action of some finite matrix group $\Gamma$ acting on $M$ (we only consider finite groups in this paper). This means that for any $x \in M$ and for any $g \in \Gamma$ we have that

$$
g f(x)=f(g x) .
$$

this will cause [8] a number of linear subspaces of $M$ to be $f$-invariant. More precisely, given any subgroup $\Sigma$ of $\Gamma$ the fixed point subspace

$$
\operatorname{Fix}(\Sigma)=\{x \in M: g x=x \text { for all } g \in \Sigma\}
$$

if $f$-invariant. Not all subgroups give rise to distinct fixed point subspaces; however those that do can be characterised by the isotropy subgroups of the action of $\Gamma$ on $M$; these are the subgroups $\Sigma$ such that there is an $x \in M$ with $\Sigma=\{g \in \Gamma: g x=x\}$; these are precisely the possible symmetries of points in $M$. We therefore obtain, for a given group action, a finite number of linear subspaces $N_{i} \subset M, i=1 \cdots n$ and isotropy subgroups $\Sigma_{i} \leq \Gamma$ such that $N_{i}=\operatorname{Fix}\left(\Sigma_{i}\right)$ and so $f\left(N_{i}\right) \subset N_{i}$. Observe that for any $N_{i}$ and $N_{j}$ then $N_{i} \cup N_{j}=N_{k}$ for some $k$ also invariant. This implies that $\Sigma_{k}$ is the smallest isotropy subgroup that contains both $\Sigma_{i}$ and $\Sigma_{j}$. Observe that $N_{i} \subset N_{j}$ if and only if $\Sigma_{i} \geq \Sigma_{j}$. Two subgroups $\Sigma_{1}, \Sigma_{2}$ are conjugate if there is a $g \in \Gamma$ such that $\Sigma_{1}=g^{-1} \Sigma_{2} g$. It is a basic result that conjugate subgroups have fixed point spaces that are mapped onto each other by the group. More precisely we have the following elementary result stated with proof for completeness.

Lemma 1 Suppose that $\Sigma$ is an isotropy subgroup and $g \in \Gamma$. Then $g^{-1} \Sigma g$ is an isotropy subgroup and Fix $\left(g^{-1} \Sigma g\right)=g^{-1}$ Fix $(\Sigma)$.

To see this, compute

$$
\begin{aligned}
\operatorname{Fix}\left(g^{-1} \Sigma g\right) & =\left\{x \in M: g^{-1} h g x=x \text { for all } h \in \Sigma\right\} \\
& =\left\{g^{-1} g x \in M: h g x=g x \text { for all } h \in \Sigma\right\}=g^{-1} \operatorname{Fix}(\Sigma) .
\end{aligned}
$$




\section{Symmetries and Lyapunov exponents}

Now suppose that we have a compact invariant set $A \subset M$. We assume that $A \subset N_{0}$ (without loss of generality). Within $N_{0}$ we assume that $A$ supports an ergodic $f$ invariant measure $\mu_{\text {nat }}$ that is a natural measure, i.e. there is a positive measure set (w.r.t. Lebesgue measure on $N_{0}$ ) such that points in these sets have ergodic averages determined by $\mu$; i.e. such that

$$
\lim _{k \rightarrow \infty} \frac{1}{k} \sum_{j=0}^{k-1} \phi\left(f^{j}(x)\right)=\int_{y} \phi(y) d \mu(y)
$$

for any continuous $\phi: N_{0} \rightarrow \mathbf{R}$ and a positive measure set of $x \in N_{0}$. We also define

$$
\operatorname{Erg}(A)=\{\mu: \text { ergodic measures supported on } A\}
$$

With respect to any ergodic measure $\mu \in \operatorname{Erg}(A)$ we define the Lyapunov exponents (L.E.s)

$$
\lambda(x, v)=\lim _{n \rightarrow \infty} \frac{1}{n} \log \frac{\left|D f^{n}(x) v\right|}{|v|}
$$

which take one of the values $\lambda_{i}(\mu), i=1, \cdots, m$ for $\mu$-almost all $x$ and any $v$. Recall that given any ergodic measure with support contained within an invariant subspace $N$, the Lyapunov exponents (L.E.s) can be split into two groups; the tangential L.E.s $\lambda_{j}^{\|}$and the transverse L.E.s $\lambda_{j}^{\perp}$ with the former corresponding to perturbations $v$ within $N$. We assume that the L.E.s are ordered greatest first; $\lambda_{1} \geq \lambda_{2}$ etc.

For nested invariant subspaces $N$ and $P$ with $A \subset N \subset P \subset M$ we define

$$
\left\{\lambda_{i}^{N, P}(\mu): i=1, \cdots, \operatorname{dim} P-\operatorname{dim} N\right\}
$$

to be the set of possible $\lambda^{\perp}(x, v)$ attained for $v \in P$ and $x$ is typical w.r.t. the measure $\mu$. We also define

$$
\Lambda_{\max }^{N, P}=\sup _{\mu \in \operatorname{Erg}(A)} \lambda_{1}^{N, P}(\mu)
$$

and

$$
\Lambda_{\text {nat }}^{N, P}=\lambda_{0}^{N, P}\left(\mu_{\text {nat }}\right) .
$$

In the case of symmetries we often obtain attractor within an invariant subspace $N$ that is nontrivially contained within several distinct invariant subspaces $P_{1}, P_{2}$ etc. The L.E.s in different directions $P_{i}$ can however in certain circumstances be related:

Theorem 1 Suppose that $N=$ Fix $(T)$ and $N \subset P_{k}=F i x\left(\Sigma_{k}\right), k=1, \cdots, l$ where $\Sigma_{k}$ are conjugate isotropy subgroups, i.e. $\Sigma_{k}=g_{k}^{-1} \Sigma_{1} g_{k}$ for some $g_{k} \in T$ and $k=2, \cdots, l$. Let $N=\cap_{k} P_{k}$ and suppose that $A \subset N$ is an attractor. Then $\lambda_{i}^{N, P_{k}}$,

$$
\Lambda_{\max }^{N, P_{k}} \text { and } \Lambda_{n a t}^{N, P_{k}}
$$

are independent of $k$. 
Proof Consider $P_{1}=\operatorname{Fix}(\Sigma)$ and $g=g_{2}$ so that $P_{2}=\operatorname{Fix}\left(g^{-1} \Sigma g\right)=g^{-1} \operatorname{Fix}(\Sigma)=$ $g^{-1} P_{1}$. Now $g x=x$ (and $|g|=1$ ) and equivariance of $f$ implies equivariance of the derivative $(D f(g x) g v=g D f(x) v)$. Hence for any $x \in N$ and $v \in \Sigma_{1}$ we have

$$
\lambda\left(g^{-1} x, g^{-1} v\right)=\lim _{n \rightarrow \infty} \frac{1}{n} \log \frac{\left|D f^{n}(g x) g v\right|}{|g v|}=\lim _{n \rightarrow \infty} \frac{1}{n} \log \frac{|g|\left|D f^{n}(x) v\right|}{|g||v|}=\lambda(x, v) .
$$

This means that any L.E. $\lambda_{i}^{N, P_{1}}$ is also a L.E. $\lambda_{i}^{N, P_{2}}$ etc.

The previous result requires that the conjugating element $g$ is in $T$. More generally we require that $g$ maps $\operatorname{Fix}(T)$ to itself which in turn implies that $g \in \operatorname{Norm}(T)$ where $\operatorname{Norm}(T)=\{h \in \Gamma: h T=T h\}$ is the normalizer of $T$. In this case the result above can be adapted if the measure $\mu_{n a t}$ has a symmetry $g$ (a symmetry on average [7]). More precisely,

Theorem 2 Suppose that $N=$ Fix $(T)$ and $N \subset P_{k}=$ Fix $\left(\Sigma_{k}\right)$ for $k=1, \cdots, l$ where $\Sigma_{k}=g_{k}^{-1} \Sigma_{0} g_{k}$ for some $g_{k} \in$ Norm $(T)$. Suppose moreover that $\mu_{n a t}$ is invariant under $g_{k}, N=\cap_{k} P_{k}$ and $A \subset N$ is an attractor. Then $\lambda_{i}^{N, P_{k}}$,

$$
\Lambda_{\max }^{N, P_{k}} \text { and } \Lambda_{n a t}^{N, P_{k}}
$$

are independent of $k$.

Proof This follows as for the previous result on noting that there is a $g_{k}$-invariant set of $x$ with full $\mu_{n a t}$-measure that has the same L.E.s. at each point.

\subsection{Directions of Riddling}

Recall that a basin of attraction $\mathcal{B}(A)$ is riddled (in $M$ ) [1] if for any $x \in A$ and $\delta>0$ we have

$$
\ell_{M}\left(\mathcal{B}(A) \cap B_{\delta}(x)\right) \ell_{M}\left(\mathcal{B}(A)^{c} \cap B_{\delta}(x)\right)>0
$$

where $\ell_{M}(\cdot)$ denotes Lebesgue measure on $M$. Similarly, we say the basin of attraction of $A \subset N_{0}$ is riddled in the direction $N_{i}$ for any invariant subspace $N_{i}$ where $N_{0} \subset N_{i}$ if

$$
\ell_{N_{i}}\left(\mathcal{B}(A) \cap B_{\delta}(x)\right) \ell_{N_{i}}\left(\mathcal{B}(A)^{c} \cap B_{\delta}(x)\right)>0 .
$$

Observe that it is necessary for $\mathcal{B}(A) \cap N_{i}$ to have positive $\ell_{N_{i}}$-measure in order to get riddling. Consider any open neighbourhood $U$ of $A$ and define the basin of $A$ relative to $U$ to be

$$
\mathcal{B}_{U}(A)=\left\{x \in M: \omega(x) \subset A \text { and } f^{n}(x) \subset U \text { for all } n \geq 0\right\} .
$$

We say the basin of $A$ is locally riddled if there is a neighbourhood $U$ of $A$ such that $\mathcal{B}_{U}(A)$ is riddled [5] (this is a stronger assumption than that given in [4]).

We say a Milnor attractor is regular if for any open neighbourhood $U$ of $A$ then $\ell_{M}\left(\mathcal{B}_{U}(A)\right)>0$; this means that a regular Milnor attractor attracts a positive 
measure set of nearby points. A result in [1] implies that any Milnor attractor that is a uniformly hyperbolic within $N$ and whose natural measure has all transverse exponents negative will be regular.

Proposition 1 Suppose that $A \subset N$ is a regular Milnor attractor that contains a periodic orbit $p$ such that $(a) W^{s}(p)$ is dense in $\mathcal{B}(A)$ and (b) $p$ has a positive transverse Lyapunov exponent and no zero Lyapunov exponents. Then the basin of $A$ is locally riddled.

Proof Consider the unstable manifold $W^{u}(p)$; this is locally an embedding of $\mathbf{R}^{k}$ where $k$ is the number of unstable eigenvalues of $p$. As this manifold is transverse to $N$ at $p$ we can find an open neighbourhood $U$ of $N$ (and hence of $A$ ) such that $W^{u}(p)$ intersects an open set $V$ that is not in $U$. Now consider $V_{n}=\bigcup_{i=0}^{n} f^{-i}(V)$; this is an open set that for all $n$ is contained in $\mathcal{B}_{U}(A)^{c}$ and such that $\overline{\cup_{n>0} V_{n}}$ contains $p$. Since it contains points transverse to $W^{u}(p)$, it must also approach preimages and points on the stable manifold of $p$ on increasing $n$. Hence we pick any $x \in \mathcal{B}(A) \cap U$ that is a point of Lebesgue density of $\mathcal{B}(A)$, and any open neighbourhood $O$ of $x$. By (a) $O$ contains a point $q$ in $W^{s}(p)$ and so $O$ must intersect $V_{N}$ in an open set $P$ for some $N$. Hence $\ell\left(\mathcal{B}_{U}(A) \cap O\right)>0$ (as $A$ is assumed regular) and $\ell\left(\mathcal{B}_{U}(A)^{c} \cap O\right)>0$ (as $\mathcal{B}_{U}(A) \supset \cup_{n>0} V_{n} \supset P$ ) and so the basin of $A$ is locally riddled.

Note that for uniformly hyperbolic $A$ (within $N$ ) all ergodic measures on $A$ are limit points of sequences of periodic measures supported within $A$ [17] and so for such sets the existence of a measure with positive transverse Lyapunov exponent implies the existence of periodic points with L.E.s that are arbitrarily close to that of the natural measure. From hereon we assume that any Milnor attractor $A$ is regular, all ergodic measures on $A$ are limits of hyperbolic periodic measures in $A$ and moreover that the stable manifolds of periodic points in $A$ are dense in $\mathcal{B}(A)$.

Remark 1 Suppose that $A$ is a Milnor attractor such that $A \subset N_{0} \subset N_{1} \subset M$. If $A$ is riddled in $M$ then it need not be riddled in either $N_{i}$. In fact the examples discussed in [1, 4] have attractors that are riddled in $M$ but asymptotically stable and therefore unriddled in the largest linear subspace $N$ that contains the attractor. What we emphasise here is that the it may be unriddled in a larger invariant subspace.

Remark 2 The same holds even if $A$ is not an attractor but a chaotic saddle in $M$. If $A$ is a Milnor attractor relative to some subspace it may or may not be riddled in that subspace.

Remark 3 Suppose that a Milnor attractor $A$ in $M$ obeys the hypotheses of Proposition 1 for the system restricted to some invariant subspace $N^{\prime}$ with $N \subset N^{\prime} \subset M$. Then the basin of $A$ is riddled in $M$. Note that the hypotheses of on $A$ with respect to the system on $N^{\prime}$ also apply to the system on the whole of $M$. We need the extra hypothesis that $A$ is an attractor in $M$ 
Remark 4 One can find systems $f: M \rightarrow M$ with an invariant set $A$ contained in an invariant subspace $N$ such that $A$ is an attractor in $M$ but not in $N$ ! For example, consider the flow induced by the vector field $(\dot{x}, \dot{y})=\left(y^{2} x-x^{3}, y^{3}-x^{2} y\right)$. This has an equilibrium at $(0,0)$ that has the open basin of attraction given by $y^{2}>x^{2}$. However, all points with $y^{2}<x^{2}$ are repelled away to infinity. Thus the origin is an attractor in $\mathbf{R}^{2}$ but has trivial basin of attraction in the invariant subspace given by the $x$-axis. In this example this is a degeneracy caused by non-hyperbolicity of the fixed point at the origin. We expect that this behaviour cannot occur in sufficiently hyperbolic systems.

\section{Anisotropic riddling and sensitivity to noise}

A traditional model for noise in iterated maps is the addition of uniformly distributed random variable at every iteration. For attractors with riddled basins, this has been recognised to give rise to discontinuous behaviour in the support of attractors as the noise goes to zero; this was called bubbling in [3]. In applications, however, the noise may be highly directional and riddled basin attractors will have a degree of sensitivity of an attractor to anisotropic noise that is dependent on whether the noise is in directions in which the basin is unriddled or not.

More precisely, suppose that $A \subset N \subset M$ with $A$ an attractor for $f: M \rightarrow M$ and the basin of $A$ is locally riddled (say $\mathcal{B}_{U}(A)$ is riddled with $U$ compact in $M$ ) but which is asymptotically stable in the invariant subspace $N$. Consider a perturbed map

$$
x_{n+1}=g\left(x_{n}, n\right)=f\left(x_{n}\right)+\sigma \xi_{n}
$$

where $\xi_{n}$ is a uniformly distributed in some compact region $R \subset M$. If $R \subset N$ and we can find an attractor $A_{\sigma}$ for (1) such that $A_{\sigma} \rightarrow A$ in the Hausdorff metric as $\sigma \rightarrow 0$. Conversely, if $R \not \subset N$ (for example if $R$ is a neighbourhood of 0 ) at each point in $A$, the noise can cause us with positive probability to exit from $\mathcal{B}_{U}(A)$. This means that $A_{\sigma} \nrightarrow A$ in the Hausdorff metric as $\sigma \rightarrow 0$ and we get bubbling of the attractor; examples of this are illustrated below.

\subsection{Internal riddling transitions}

Given any attractor $A$ with a riddled basin $\mathcal{B}(A)$ we characterise this basin by examining the dimension $\tilde{d}$ of the largest invariant subspace $N$ containing $A$ such that the basin of $\mathcal{B}(A) \cap N$ is unriddled. Note that $\operatorname{dim}(A) \leq \tilde{d} \leq \operatorname{dim}(M)$. Any change in $\tilde{d}$ we term an internal riddling transition. It is clear that such transitions will occur in higher-dimensional systems; as $A$ loses asymptotic stability in more directions the index $\tilde{d}$ will decrease. Similarly one can apply the standard riddling bifurcation criteria in each subspace to predict parameter values when internal riddling transitions will occur; see $[5,10,11,14,19]$. Such transitions will typically be rather unclear on varying system parameters if the chaotic attractors are not structurally stable; only by examining normal parameters of the system such that the dynamics on the attractor is left unchanged can one hope to find internal riddling transitions 
appearing as codimension one transitions. Similar transitions where riddling bifurcations are replaced by blowout bifurcations will also occur in such systems; see for example the systems studied in [2].

\subsection{A numerical example}

We now examine an example mapping where we can investigate the effects of riddling in different directions. Consider the map on $\mathbf{R}^{4}$ defined by

$$
x_{i}^{\prime}=(1-\epsilon) f\left(x_{i}\right)+\frac{\epsilon}{n} \sum_{j=1}^{n} f\left(x_{j}\right)+\sigma_{i} \eta_{i}
$$

for $i=1, \cdots, 4$, where at each timestep each $\eta_{j}$ is an independent random variable that is uniformly distributed on $-[0.5,0.5]$. The noise in the $i$ th component has amplitude that can be controlled with $\sigma_{i}$. This map has been studied by several authors including Kaneko [9], although the only noise perturbations considered have been isotropic. Note also that Taborev et al [18] have recently looked at this system with 3 cells in some detail.

This map has the symmetry of all permutations on $n$ objects; in this case $S_{4}$. There are a large number of invariant subspaces corresponding to isotropy subgroups that can be characterised by partitions of $\{1 . .4\}$ into $1 \leq m \leq 4$ groups of identical cells, namely all subgroups of the form $S_{1}^{4}, S_{2} \times S_{1}^{2}, S_{2} \times S_{2}, S_{3} \times S_{1}$ and $S_{4}$.

In such a system at parameter values that are intermediate between strong and weak coupling one may find a wide variety of attractors of different symmetries that are multi-stable. One can also find, for example, (i) chaotic saddles that have basins that are riddled within certain invariant subspaces (ii) attractors that are riddled in some directions but not others and hence (iii) anisotropic bubbling response to anisotropic noise.

As a specific example we consider an attractor in

$$
\operatorname{Fix}\left(S_{2} \times S_{2}\right)=\{(p, p, q, q): p, q \in \mathbf{R}\} .
$$

that occurs when $a=1.71$ and $\epsilon=0.15$. Figure 1(i) shows a timeseries on this attractor for an initial condition very close to $(0.00292,0.00292,0.8004,0.8004)$ and no added noise; $\sigma_{j}=0$ for all $j$. On addition of noise (iia) shows $x_{1}-x_{2}$ for the same initial condition but with $\sigma_{1}=10^{-5}$. The large deviations away from $x_{1}=x_{2}$ indicative of bubbling in the direction $(1,0,0,0)$ and hence indicate that the basin of attraction of this attractor is riddled to perturbations into the invariant subspace $(p, q, r, r)$. By contrast, (iib) shows $x_{3}-x_{4}$ for for the same initial condition but with $\sigma_{3}=10^{-5}$. In this case there is apparently stable response indicating that the basin is not riddled into the invariant subspace $(p, p, q, r)$. Note that (i) shows that the statistics of $x_{1}$ and $x_{3}$ are quite different; the natural invariant measure associated with this attractor is not invariant under the transformation

$$
\left(x_{1}, x_{2}, x_{3}, x_{4}\right) \mapsto\left(x_{3}, x_{4}, x_{1}, x_{2}\right) .
$$

If this symmetry did leave the attractor invariant then by applying Theorem 2 either both or neither of the directions $(1,0,0,0)$ and $(0,0,1,0)$ would be riddled. 
Numerical simulations indicate that the transverse L.E. in the direction $(1,0,0,0)$ is approximately $-7.8 \times 10^{-4}$ whereas it is approximately -0.2842 in the direction $(0,0,1,0)$; this agrees with the anisotropic bubbling observations.

\subsection{Discussion}

In this paper we demonstrate that the presence of basins of attraction that are riddled in some directions but not in others is in fact typical in higher dimensional systems. This can give rise to a number of new phenomena that will be typical in such symmetric systems.

This anisotropic riddling in only certain directions leads to sensitivity under perturbation that can vary a lot between different directions. Work in progress [6] indicates this gives a good model for the sensitivity of neural systems to minute inputs, for example allowing the brain to classify smells given very minor inputs. Note that basin riddling has already been found in the learning dynamics of neural networks [13].

\section{References}

[1] Alexander, J.C., Yorke, J.A. You, Z. and Kan, I., Riddled Basins. Int. J. Bif. Chaos 2, 795-813 (1992).

[2] Ashwin, P. and Aston, P.J. Blowout bifurcations of codimension two. Phys. Lett. A 244, 261-270 (1998).

[3] Ashwin, P., Buescu, J. and Stewart, I., Bubbling of attractors and synchronisation of oscillators. Phys. Lett. A 193, 126-139 (1994).

[4] Ashwin, P., Buescu, J. and Stewart, I., From attractor to chaotic saddle: a tale of transverse instability. Nonlinearity 9, 703-737 (1996).

[5] Ashwin, P. and Terry, J.R., On riddling and weak attractors. Physica D 142, 87-100 (2000).

[6] Breakspear, M., A model of sensory perception in an ensemble of globally coupled neurons, (in preparation, 2000).

[7] Dellnitz, M., Golubitsky, M., Nicol, M., Symmetry of attractors and the Karhunen-Love decomposition. Trends and perspectives in applied mathematics pp 73-108, Appl. Math. Sci., 100, Springer, New York, (1994).

[8] Golubitsky M., Stewart I.N., and Schaeffer, D., Groups and Singularities in Bifurcation Theory. Volume 2. Appl. Math. Sci. 69, Springer-Verlag, New York, (1988).

[9] Kaneko, K., On the strength of attractors in a high-dimensional system: Milnor attractor network, robust global attraction, and noise-induced selection. Physica D 124, 322-344 (1998). 


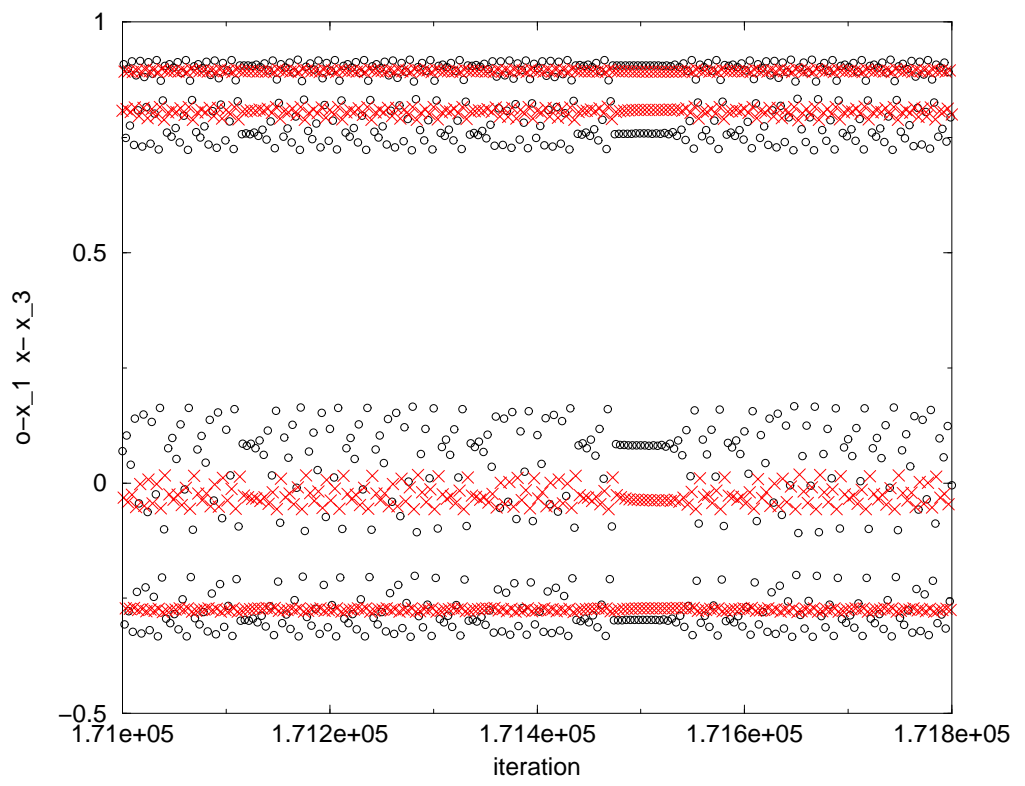

(i)
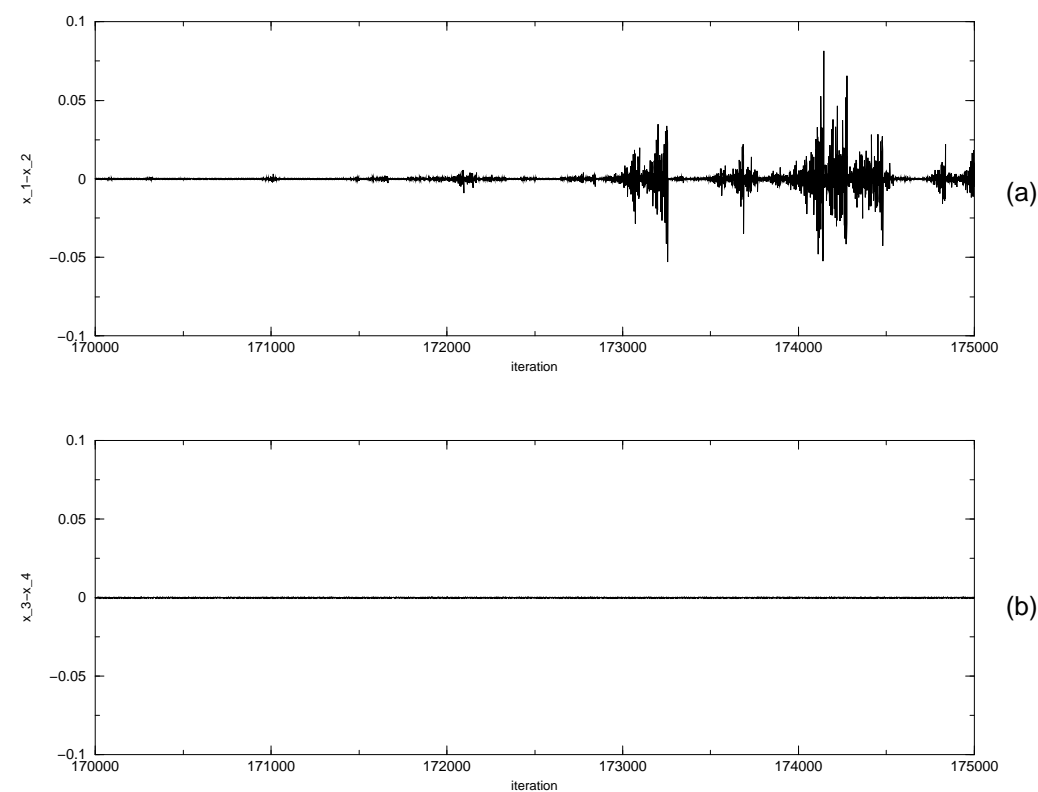

(ii)

Figure 1: Anisotropic bubbling behaviour caused by anisotropic riddling of the basin of an attractor for a system of 4 globally coupled maps (2). (i) shows timeseries of response of the noise-free system; the circles show the values of $x_{1}$ and $x_{2}$ while the crosses show the values of $x_{3}$ and $x_{4}$. In (ii) the attractor in (i) is subject to very low amplitude noise. In (iia) the noise is added in the $x_{1}$ direction only giving rise to bubbling in this direction. In (iib) it is added in the $x_{3}$ direction giving a stable response, due to the attractor not being riddled in this direction. 
[10] Lai, Y-C., Grebogi, C., Yorke, J.A., and Venkataramani, S.C., Riddling bifurcation in chaotic dynamical systems. Phys. Rev. Lett. 77, 55-58 (1996).

[11] Maistrenko, Yu. L., Maistrenko, V. L., Popovich, A. and Mosekilde, E., Transverse instability and riddled basins in a system of two coupled logistic maps. Phys. Rev. E 57, 2713-2724 (1998).

[12] Milnor, J., On the concept of attractor. Commun. Math. Phys. 99, 177-195 (1985); Comments Commun. Math. Phys. 102, 517-519 (1985).

[13] Nakajima, H. and Ueda, Y., Riddled basins of the optimal states in learning dynamical systems. Physica D 99, 35-44 (1996).

[14] Ott, E., Sommerer, J.C., Alexander, J., Kan, I. and Yorke, J.A., A transition to chaotic attractors with riddled basins. Physica D 76, 384-410 (1994).

[15] Ott, E. and Sommerer, J., Blowout bifurcations: the occurrence of riddled basins and on-off intermittency. Phys. Lett. A 188, 39-47 (1994).

[16] Pikovsky, A.S. and Grassberger, P., Symmetry breaking of coupled chaotic attractors. J. Phys. A, 24, 4587-4597, (1991).

[17] Sygmund, K. Generic properties of invariant measures for Axiom A diffeomorphisms. Inv. Math. 11, 99-109, (1970).

[18] Taborev, A.V., Maistrenko, Yu. L. and Mosekilde, E. Partial synchronization in a system of coupled logistic maps. Int. J. Bifurcation and Chaos 10, 1051-1066 (2000).

[19] Venkataramani, S.C., Hunt, B.R., Ott, E., Gauthier, D.J. and Bienfang, J.C., Transitions to bubbling of chaotic systems. Phys. Rev. Lett. 77, 5361-5364 (1996).

[20] Yamada, H. and Fujisaka, T., Stability theory of synchronised motion in coupled-oscillator systems. Prog. Theor. Phys. 70, 1240-1248 (1984). 